\title{
Improving and testing the empirical equatorial electrojet model with CHAMP satellite data
}

\author{
V. Doumouya ${ }^{1}$ and Y. Cohen ${ }^{2}$ \\ ${ }^{1}$ Laboratoire de Physique de l'Atmosphère, Université de Cocody, République de Côte d'Ivoire \\ ${ }^{2}$ Dépt. de Géophysique Spatiale et Planétaire, Inst. de Physique du Globe de Paris, 94107 Saint-Maur, France \\ Received: 4 October 2003 - Revised: 7 July 2004 - Accepted: 12 July 2004 - Published: 23 September 2004 \\ Part of Special Issue "Equatorial and low latitude aeronomy"
}

\begin{abstract}
The longitudinal variation of the Equatorial Electrojet (EEJ) intensity has been revised including data from the equatorial station of Baclieu (Vietnam), where an unexpected enhancement of the EEJ magnetic effects is observed. The features of this longitudinal variation were also obtained with the CHAMP satellite, except in the Pacific and Atlantic Oceans, where no ground level data points were available.The EEJ magnetic signatures recorded on board the CHAMP satellite have been isolated for 325 passes in different longitude sectors around local noon. The results have been compared with the EEJ magnetic effects computed using the Empirical Equatorial Electrojet Model (3EM) proposed by Doumouya et al. (2003). The modeled EEJ magnetic effects are generally in good agreement with CHAMP observed EEJ magnetic signatures.
\end{abstract}

Key words. Ionosphere (Equatorial ionosphere; Electric fields and currents)

\section{Introduction}

The Equatorial Electrojet (EEJ) is a dayside ionospheric current sheet, flowing eastward along the dip-equator, at an altitude of about $105 \mathrm{~km}$. This intense current system was discovered in 1922, following the setup of the geomagnetic observatory of Huancayo in Peru (Forbush and Casaverde, 1961).

The magnetic signature of the EEJ have been evidenced in the high altitude geomagnetic data recorded by the Polar Orbiting Geomagnetic Observatories (POGO) series of spacecrafts, by a sharp depression at the dip-equator (Cain and Sweeney, 1973). The different features of the EEJ have been investigated through more than 2000 profiles (Cain and Sweeney, 1973; Onwumechili and Agu, 1980; Agu and Onwumechili, 1981). The POGO data have also been exploited to seek correlations between surface and satellite borne EEJ

Correspondence to: V. Doumouya

(doumouya@ipgp.jussieu.fr) magnetic signatures (Cain and Sweeney, 1973; Osborne, 1973; Ebun Oni, 1973; Yacob and Bhargava, 1973; Kane, 1973; Gouin, 1973; Agu and Onwumechili, 1981). Despite the dawn-dusk orbit of the Magsat satellite, the magnetic signature of the EEJ revealed significant amplitude that affected the anomaly maps deduced from Magsat magnetic data (Langel et al., 1982; Cohen and Achache, 1990; Langel et al., 1993). The influences of the EEJ in spatial geomagnetic measurements have been widely mentioned during the Magsat data analysis (Yanagisawa and Kono, 1985; Cohen and Achache, 1990; Langel et al., 1993; Ravat and Hinze, 1993).

In a global sense, the magnetic field recorded on board low altitude satellites includes internal fields that originate in the Earth's interior (the main field and the crustal fields), and external fields that are associated with the ionospheric and magnetospheric current systems. Thus, each of those contributions could be separately studied through satellite data. But, their identification and separation are still difficult due to the overlapping of internal and external fields. In a recent work, Sabaka et al. (2002) proposed a global comprehensive model, designated CM3, which takes into account various contributions. In this model, only an averaged consideration is taken of the ionospheric current contributions. Such an approach would not specifically take into account the EEJ contribution. Due to the limited extent of its magnetic effects in latitudes, the EEJ should be considered as a necessary part of global models.

Doumouya et al. (2003) proposed an empirical model of the EEJ, using ground-based magnetic data, recorded in a network of 26 stations during the International Equatorial Electrojet Year (Amory-Mazaudier et al., 1993; Arora et al., 1993; Rigoti et al., 1999). In this paper, The EEJ contribution to the CHAMP satellite magnetic measurements will be studied and be used to evaluate the Empirical Equatorial Electrojet Model proposed by Doumouya et al. (2003), referred to as "3EM". 


\section{The magnetic signature of the equatorial electrojet onboard CHAMP satellite}

The CHAMP satellite records the Earth's magnetic field at an altitude of $450 \mathrm{~km}$ (Lühr et al., 2002), sweeping different local times. Thus, the magnetic data of the CHAMP satellite are well designed for studying the daytime ionospheric current systems (e.g. the EEJ) through their associated magnetic variations. For this study, we have been provided with 7 months of data, from June to December 2001. The satellite daily passes crossing the dip-equator, along the local noon, between August and September, 2001, are employed. Among 896 daily passes, 325 belong to the time interval of 11:00 to 13:00 LT.

\subsection{Data processing}

The main purpose of this paper is to evaluate the 3EM proposed by Doumouya et al. (2003) against the CHAMP satellite borne EEJ magnetic signature. This work requires a preliminary separation of the EEJ magnetic effects from fields of other sources. The amplitude of the EEJ magnetic signature is very small with respect to that of the main field. This fact makes it necessary to remove an estimate of the main field from the total field measurements. Hence, the main field is subtracted using the IGRF 2000 model for the "epoch 2001.5". The strong amplitude (about $100 \mathrm{nT}$ ) of the remaining residuals (Fig. 1) implies that the main field could have not been totally removed. It implies that the IGRF field represented by spherical harmonics of degree and order 10 does not account for the total intensity of the main field. Langel and Estes (1982), and Cohen and Achache (1990) suggested a main field model of degree and order 13 for a better approximation of the main field intensity. In a more recent work, Maus et al. (2002) used a main field model of degree and order extended to 29 to remove the main field from CHAMP scalar data. It is possible that a main field model of such a high degree and order may remove a significant part of the ionospheric field itself, especially that of the EEJ.

The residual field in Fig. 1 results from the overlapping components from the Earth's crust, the magnetosphere and the ionosphere. Since the EEJ associated magnetic effects extend only over a narrow latitude band across the dipequator, they are assumed to be superimposed over a very regularly shaped background field. The long wavelength background field has been simply extrapolated under the EEJ influence area, using a polynomial fitting. A similar approach based on linear extrapolation was used to isolate the POGO EEJ signature (Cain and Sweeney, 1973; Onwumechili and Agu, 1980). Langel et al. (1993) isolated the dip-latitude dependent fields using a Kaiser pass-by-pass filter. Notice that the polynomial fitting of the background field has not any physical basis. Furthermore, the corresponding EEJ signature depends on the degree of the polynomial, as well as on the latitude extent of the EEJ signature.
2.2 Determination of the EEJ peak $\Delta F$ from CHAMP scalar magnetic data

The EEJ is a narrow current sheet flowing in the dayside ionosphere, at the altitude of about $105 \mathrm{~km}$, with a total width of about $7^{\circ}$ geomagnetic latitude (Fambitakoye and Mayaud, 1976a; Doumouya et al., 1998). Its satellite borne magnetic effects similarly expand on a relatively wider latitude band, marking a quasi-sharp depression with a peak value at the dip-equator, and sometimes two shoulders on either side. Figure 2 presents the averaged absolute values of the CHAMP noon EEJ magnetic signature across the dipequator, based on August and September 2001 data. The amplitude $(\Delta F)$ of the EEJ associated magnetic signatures is determined at the EEJ axis from CHAMP latitude profiles as the difference between the minimum peak and the average of the shoulders. About 325 values of $\Delta F$ have been determined around noon during August and September 2001. These values are used to establish the CHAMP borne longitude profile (Fig. 3) of the EEJ magnetic effects along the dip equator. The dotted plot represents the individual $\Delta F$ versus the longitude of each satellite pass, whereas the blue line curves depict average behavior. In Fig. 4, we have reproduced the longitudinal profile of EEJ, obtained earlier by Doumouya et al. (2003) using the IEEY ground-based variation of EEJ $\Delta H$ peak values around noon. It is expected that despite the difference in epoch of field data, both satellite and ground-based plots should highlight the salient features of the longitudinal variability in EEJ. However, the compassion of longitudinal profiles of EEJ in Fig. 3 (satellite-based) and Fig. 4 (groundbased) show broad similarity, though some differences, particularly in the longitudinal sector of $100^{\circ} \mathrm{E}$, are distinctly seen. The longitudinal profile resulting from satellite data shows a strong enhancement of the EEJ magnetic effect in the longitude sector of Vietnam. It may be noted that the longitude profile of $\Delta H$ (Fig. 4) presented by Doumouya et al. (2003) might not have taken into account some local features due to the small number of data points, controlled by the distribution of ground-based observatories. It should be mentioned that there were no observations neither in the Atlantic nor in the Pacific Oceans, as well as around $100^{\circ} \mathrm{E}$. The lack of data in the longitude sector around $100^{\circ} \mathrm{E}$ can be filled by the data now available from the equatorial station of Baclieu (Vietnam). The revised longitudinal plot, obtained by the inclusions of Baclieu data, is included in Fig. 3 and Fig. 4 (green line). The strong enhancements of EEJ in South America and around $100^{\circ} \mathrm{E}$ (Vietnam) are shown by the revised ground- as well as the satellite-based longitudinal plots.

\section{Using the CHAMP EEJ mean longitudinal variation with the 3EM}

Doumouya et al. (2003) have presented an empirical model of the EEJ based on surface magnetic measurements involving a network of 26 equatorial stations that operated during 

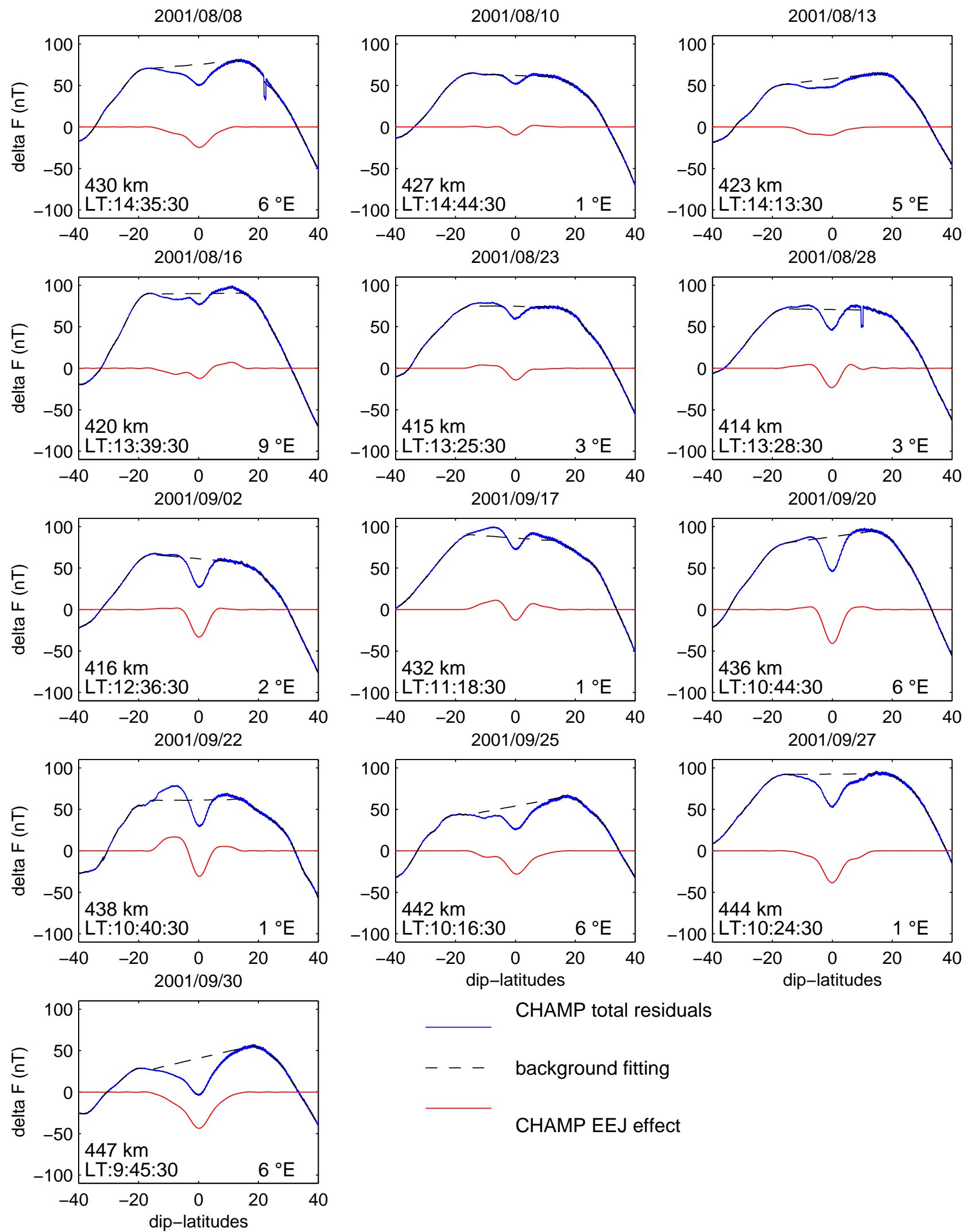

CHAMP total residuals

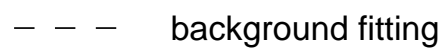

\section{CHAMP EEJ effect}

Fig. 1. Isolation of the EEJ magnetic signature from CHAMP satellite total residuals. The total magnetic residuals (blue solid lines) from CHAMP data have been extracted from the total field using the main field IGRF2000 model for the epoch 2001.5. To isolate the EEJ effect (red solid line), the background shape has been fitted using a polynomial function (dashed black line), and subtracted from the total residual. 


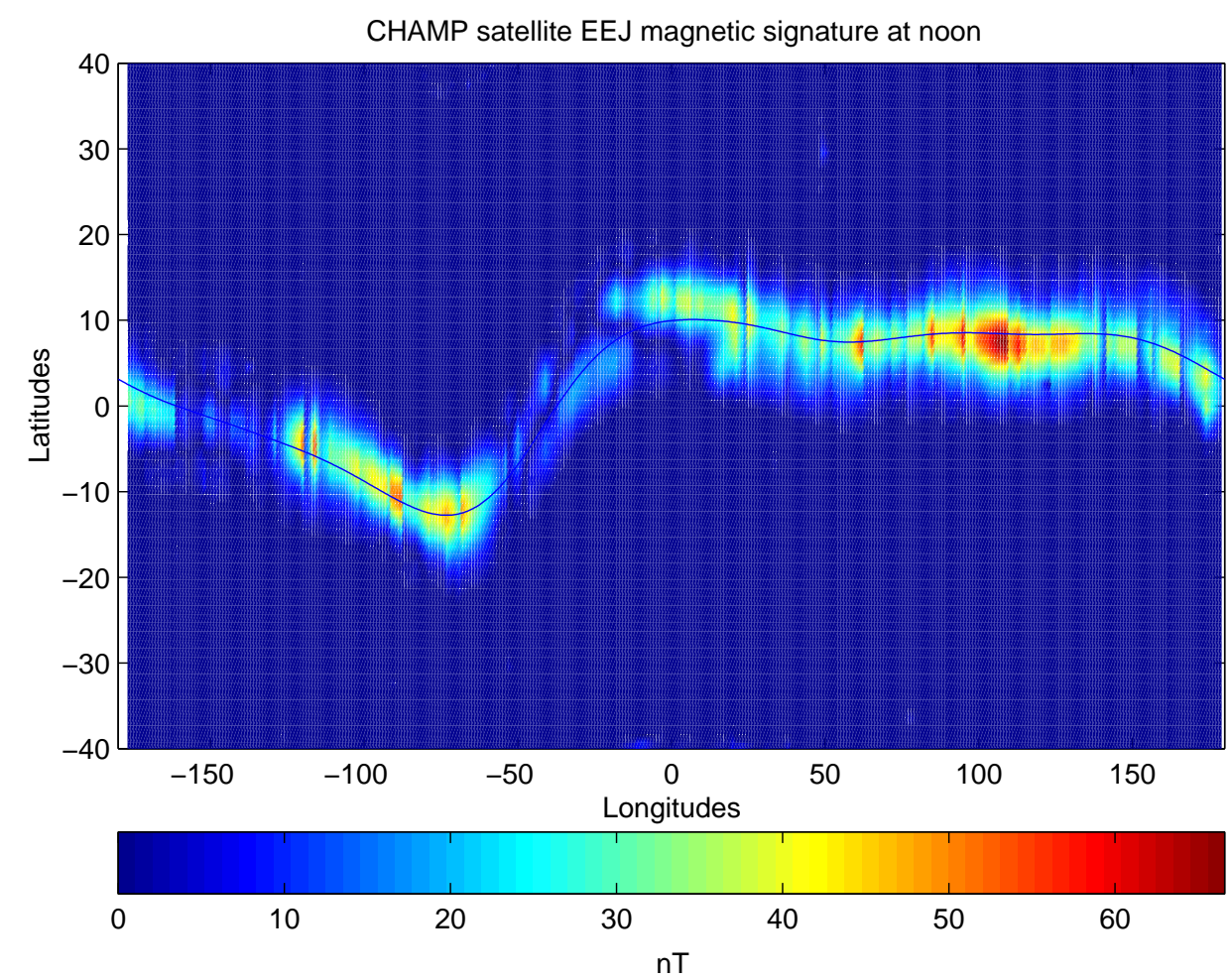

Fig. 2. CHAMP satellite noon EEJ magnetic signature in August and September 2001. This plot represents the absolute values of the EEJ magnetic effects along the dip-equator.

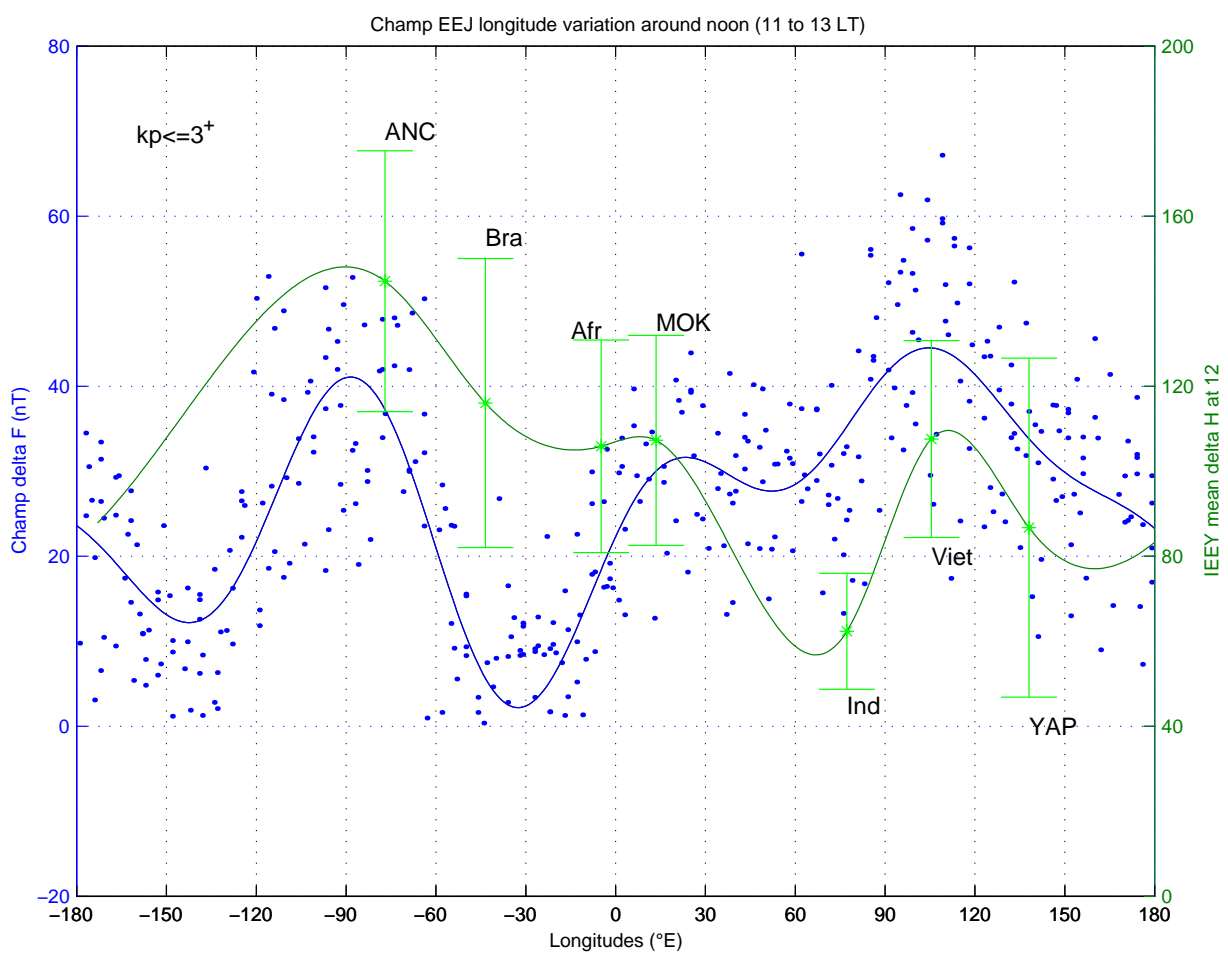

Fig. 3. The longitudinal variation of CHAMP satellite borne EEJ magnetic signature around noon (from 11:00 to 13:00 LT). The dotted plot represents the individual $\Delta F$ versus the longitude of each satellite pass, whereas the blue line curves depict average behavior. The green line with error bars represents the ground-based mean longitudinal variation of the EEJ magnetic effects at $12 \mathrm{H}$, including data from Baclieu (Vietnam). 


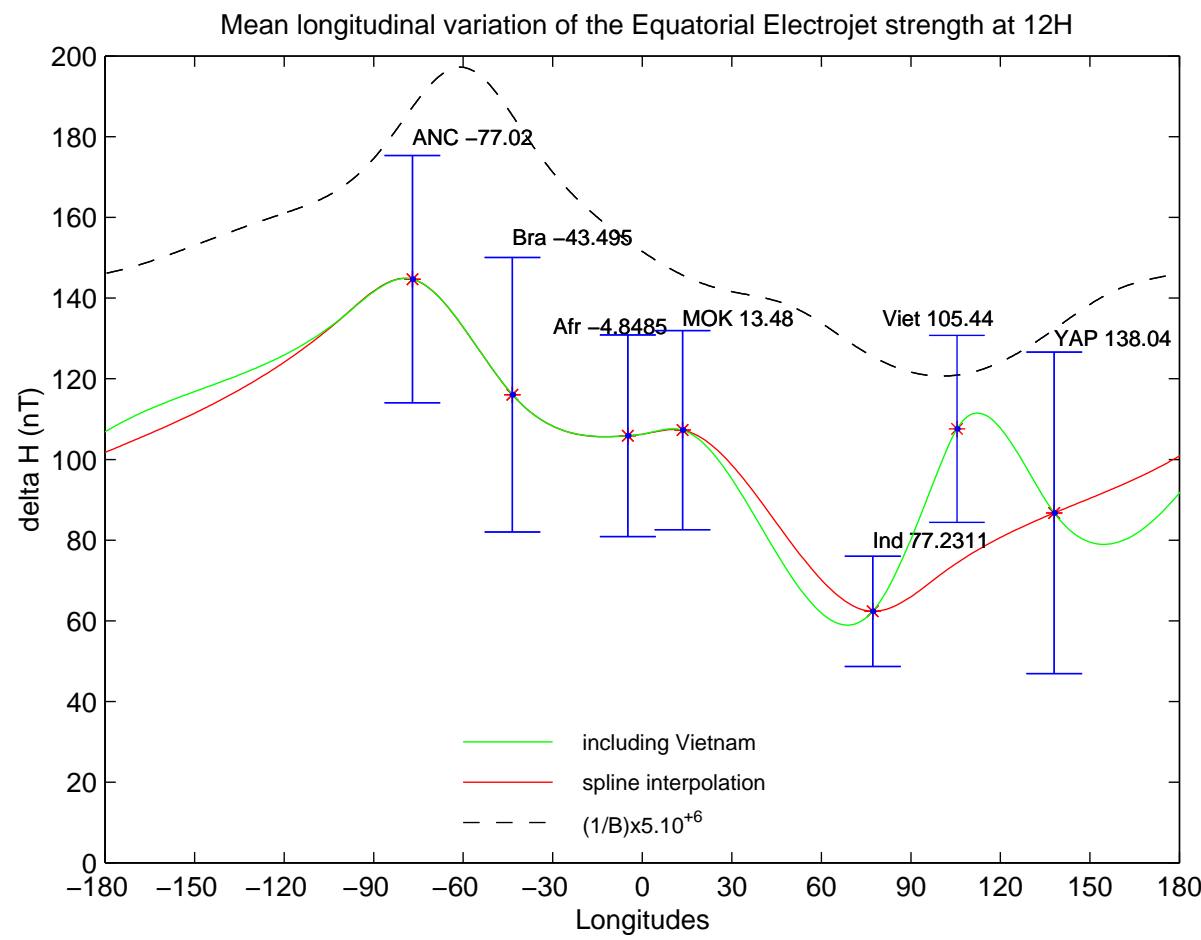

Fig. 4. The mean longitudinal variation of the EEJ magnetic effects (solid lines) from IEEY ground-based magnetic data at $12 \mathrm{~h}$, and the inverse of the main field 1/B exaggerated $5 \times 10^{6}$ times (dashed line). The green line (same as in Fig. 3) represents the ground-based longitudinal variation of the EEJ magnetic effects at $12 \mathrm{H}$, including data from Baclieu (Vietnam), while the red curve does not take data from Baclieu into account. The error bars represent the standard deviations of $\Delta H$. The curve 1/B is obtained at regular interval in longitude.

the International Equatorial Electrojet Year (IEEY). That model, referred to as "3EM", includes the local time, latitude and longitude dependences. The local time variation of the horizontal component of the EEJ magnetic effect was described with a Gaussian-like function:

$G(t)=\exp \left(-\frac{(t-T)^{2}}{t_{m}^{2}}\right)$

where $T$ is the local hour of the maximum of $\Delta H$ in the daily variation under the EEJ, $T=12: 00 \mathrm{LT}$ on average; $t_{m}$ is a fitting parameter that controls the time interval of the Gaussian-like shape of the diurnal variation in $\Delta H$ and $\Delta Z$, $t_{m}=4 \mathrm{~h}$.

The latitudinal variation was described by the "quadratic" current distribution of Fambitakoye and Mayaud (1976) with a current intensity $\left(I_{0}\right)$ at the EEJ center:

$I(x)=I_{0}\left(1-\frac{(x-c)^{2}}{a^{2}}\right)^{2}$,

Where $\mathrm{x}$ is the northward distance from the EEJ center and is defined as: $c-a<x \leq c+a ; a$ is the half-width and $c$ the position of the EEJ center. The current intensity $\left(I_{0}\right)$ at the center of the EEJ was determined through the expression:

$I_{0}=\frac{\Delta H_{0}}{0.4 \operatorname{arctg}\left(\frac{a}{h}\right)}$

(Doumouya et al., 1998; Doumouya et al., 2003).
The EEJ current distribution was then represented by the following expression:

$I(t, x, \lambda)=I o(\lambda) \cdot\left(1-\frac{(x-c)^{2}}{a^{2}}\right)^{2} \cdot \exp \left(-\frac{(t-T)^{2}}{t_{m}^{2}}\right)$

where $I_{0}(\lambda)$ represents the current intensity along the EEJ axis. Its longitudinal variation was studied through the mean longitude profile of $\Delta H$ that is related to $I_{0}$ through the Eq. (3) at a given longitude. For this study, the CHAMP satellite borne mean longitudinal profile of $\Delta F$ is used to compute the model EEJ magnetic effects.

\section{Results of the model at CHAMP altitude and epoch}

The CHAMP mean longitudinal profile of $\Delta F$ along the EEJ axis has been used in the model to compute the EEJ magnetic signature at CHAMP altitude. Thus, the model estimates the satellite borne EEJ magnetic signature at all local times, latitudes and longitudes for a given universal time. Figures 5a,b and show the snapshots of the horizontal $(H)$ and vertical $(\mathrm{Z})$ components of the EEJ magnetic effects at $450 \mathrm{~km}$ altitude, respectively, for 00:00 UT and 11:00 UT. Each CHAMP onboard latitude profile of the EEJ signature is assumed to correspond to a single meridian traverse through these structures. Thus, taking into account the mean longitudes, the local times and the altitudes of CHAMP orbits, we 

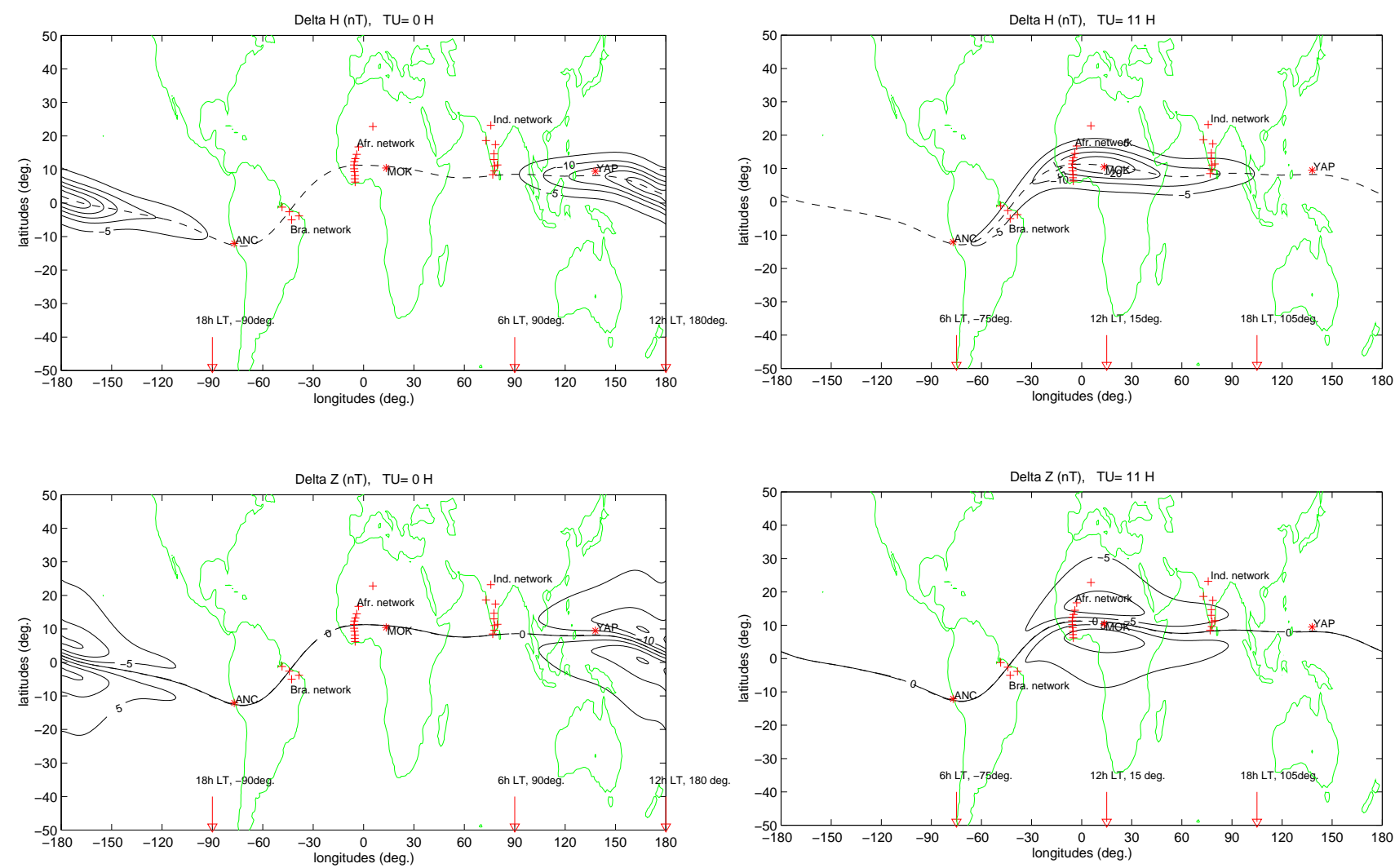

Fig. 5. (a) A snapshot of the $H$ and $Z$ components of the EEJ model magnetic signature at a fixed universal time (00:00 UT) and an altitude of $450 \mathrm{~km}$. The CHAMP satellite mean longitudinal variation was used to compute the EEJ magnetic signature at CHAMP altitude and epoch. $\Delta H$ contour interval is $5 \mathrm{nT}$ for a minimum of about $-30 \mathrm{nT}, \Delta Z$ contour interval is $5 \mathrm{nT}$ with a minimum of $-15 \mathrm{nT}$ and a maximum of $15 \mathrm{nT}$. The dashed line represents the dip-equator.

(b) A snapshot of the $H$ and $Z$ components of the EEJ model magnetic signature at a fixed universal time (11:00 UT) and an altitude of $450 \mathrm{~km}$. The CHAMP satellite mean longitudinal variation was used to compute the EEJ magnetic signature at CHAMP altitude and epoch. $\Delta H$ contour interval is $5 \mathrm{nT}$ for a minimum of about $-30 \mathrm{nT}, \Delta Z$ contour interval is $5 \mathrm{nT}$ with a minimum of $-15 \mathrm{nT}$ and a maximum of $15 \mathrm{nT}$. The dashed line represents the dip-equator.

compute the corresponding modeled EEJ $\Delta F$ latitude profiles. In the next section, the resulting latitude profiles of the EEJ magnetic signature will be compared with the corresponding CHAMP satellite recorded profiles.

\section{Comparison between the model and the CHAMP satellite EEJ magnetic effects in different longitude sectors}

This section is aiming to evaluate the model efficiency in reproducing the EEJ magnetic effects recorded by the CHAMP satellite. This evaluation is performed using two different approaches. The first one consists in extrapolating groundbased EEJ magnetic effects observed in the West African network to the altitude of an overhead CHAMP traverse. Notice that a chain of three magnetic stations has been installed across the dip-equator in West Africa (Côte d'Ivoire and Mali, $5^{\circ} \mathrm{W}$ ), since 1998, in order to record the EEJ magnetic effects simultaneously with $\phi$ rsted and CHAMP satellites.
Data from this network are used to deduce the EEJ parameters, especially the current intensity $I_{0}$ at the center (Fambitakoye and Mayaud, 1976a; Doumouya et al., 1998). Since the individual daily parameters are used, this process takes into account the EEJ day-to-day variability. The groundbased EEJ parameters are used as input in the model to estimate the EEJ magnetic signature, taking into account the corresponding CHAMP orbit parameters (date, local time, altitude, longitude). Figure 6 shows 13 diagrams where the modeled and CHAMP borne recorded EEJ magnetic signatures are plotted for 13 selected passes above West Africa. In each diagram, the dates, local times, longitudes, altitudes and the root mean squares (rms) are indicated. Except for 17 September 2001 and 25 September 2001, when strong discrepancies of $32 \mathrm{nT}$ and $42 \mathrm{nT}$ are observed (the rms during these days are $5 \mathrm{nT}$ and $6 \mathrm{nT}$, respectively), the CHAMP satellite borne EEJ signatures and the modeled signatures are generally in good agreement. The rms are most of the time less than $3 \mathrm{nT}$, corresponding to an accuracy less than $10 \%$ with respect to the peak amplitudes of measured signatures. 


\section{Comparison between the EEJ model and Champ board EEJ signatures}
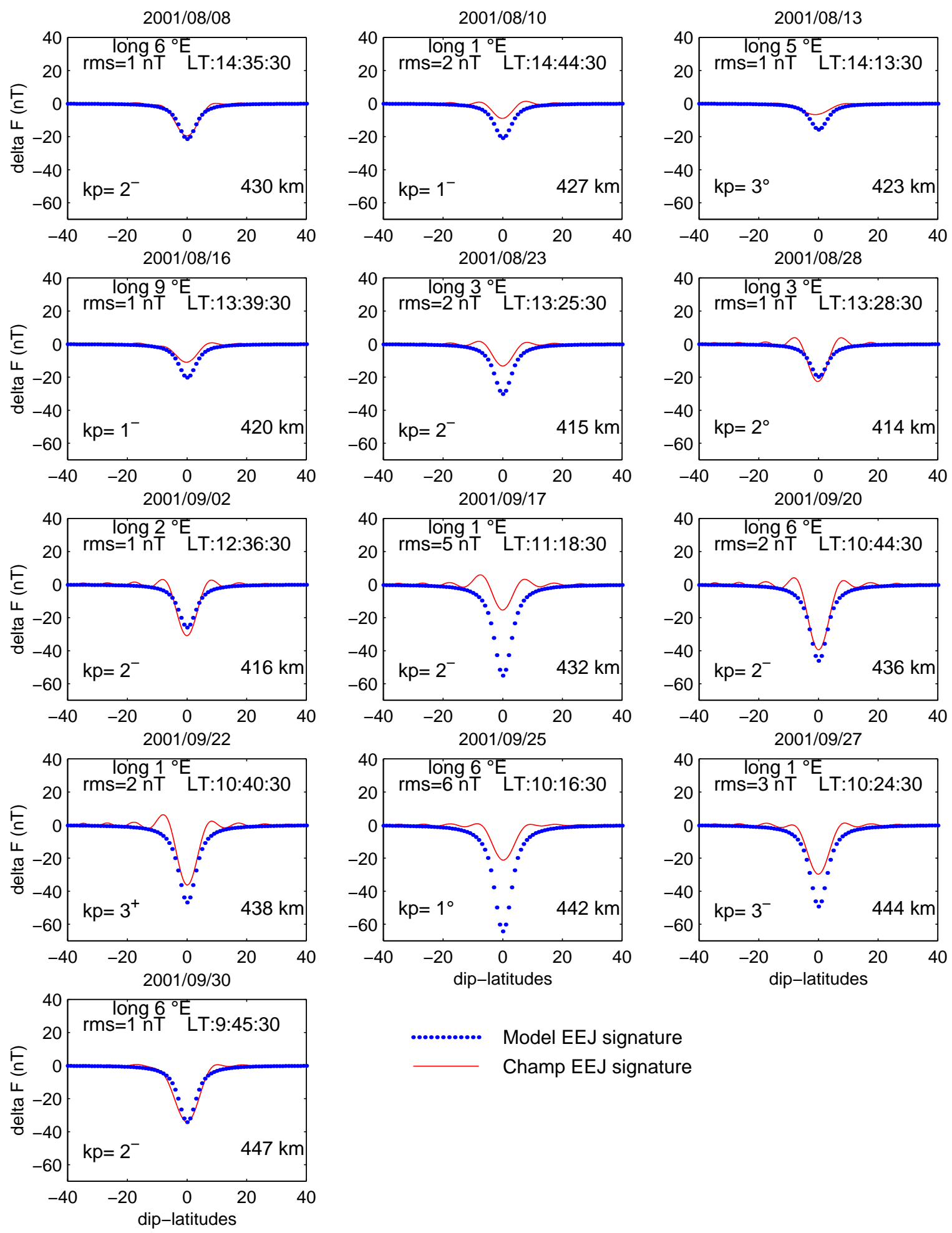

Fig. 6. Comparison between the CHAMP satellite magnetic signature and the modeled magnetic effects of the EEJ. The 3EM magnetic effects extrapolated at CHAMP satellite altitude (blue dotted line) are compared with the overhead CHAMP EEJ signatures (red solid line). The modeled profiles were computed using ground-based EEJ parameters deduced from data recorded in West Africa. The altitude, longitude, local time and the rms are indicated. 


\section{Comparison between the EEJ model and Champ board EEJ signatures}
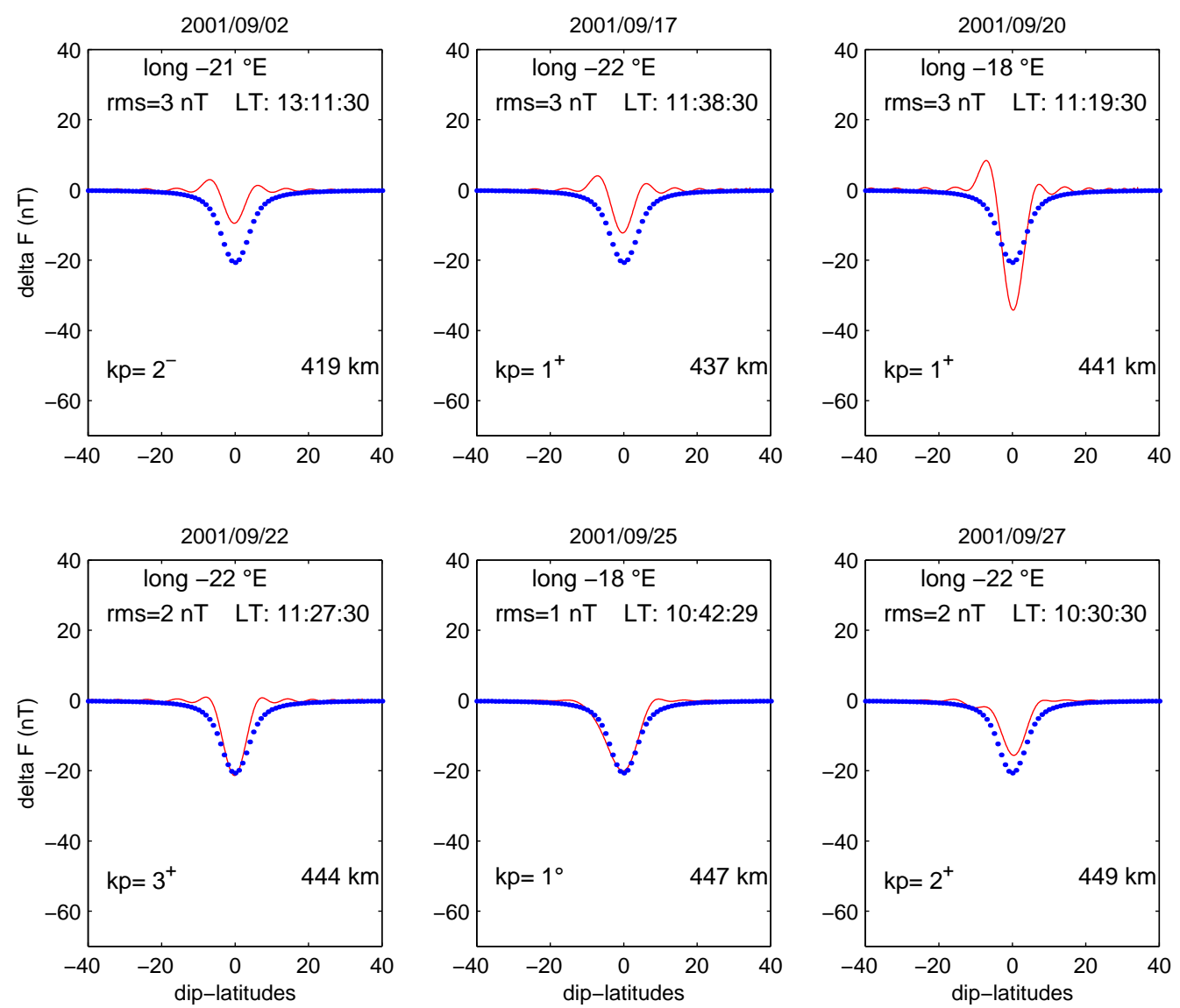

Model EEJ signature

Champ EEJ signature

Fig. 7a. Comparison between the CHAMP satellite magnetic signature and the modeled magnetic effects of the EEJ. The modeled EEJ magnetic effects extrapolated at the altitude, longitude and local time of CHAMP satellite passes (blue dotted line) are compared with the observed EEJ signatures (red solid line) in the longitude band $15^{\circ} \mathrm{W}$ to $30^{\circ} \mathrm{W}$. The modeled profiles were computed using the mean longitudinal profile of the EEJ magnetic signature. The altitude, longitude, local time and the rms are indicated.

The second approach consists in evaluating the model in other longitude sectors. Thus, the EEJ signatures are estimated for the selected longitude sectors, taking into account the orbit parameters of the corresponding satellite profiles. In Figs. 7a,b,c, the estimated EEJ signatures are compared with the satellite observations, respectively, in the longitude bands $15^{\circ} \mathrm{W}-30^{\circ} \mathrm{W}, 45^{\circ} \mathrm{E}-60^{\circ} \mathrm{E}$ and $90^{\circ} \mathrm{E}-105^{\circ} \mathrm{E}$. In all these sectors the model reproduces quite well the CHAMP satellite EEJ magnetic signature, since the differences between model and data are still less than $10 \mathrm{nT}$. By the way, notice that this second approach, based on CHAMP mean longitude variation does not take into account the day-to-day variability.

\section{Discussion and conclusions}

In this work, the longitudinal variation of the EEJ intensity has been revised, including further ground data, especially from the equatorial station of Baclieu (Vietnam), where an enhancement of the EEJ $\Delta H$ has been observed. This local feature of the EEJ in the longitude sector around $100^{\circ} \mathrm{E}$, also shown by the CHAMP satellite longitudinal variation, was not taken into account by Doumouya et al. (2003). Notice that the correlation, shown by Doumouya et al. (2003), between the EEJ $\Delta H$ and the inverse main field intensity $(1 / \mathrm{B})$, is not verified along the Vietnam longitude sector. The origin of that unexpected enhancement in the EEJ longitudinal variation is unknown, since the ambient internal geomagnetic field intensity is stronger (about $45000 \mathrm{nT}$ ). It could be related to a particular feature of the ionospheric dynamo parameters, such as the neutral wind, the conductivities, or the 
Comparison between the EEJ model and Champ board EEJ signatures
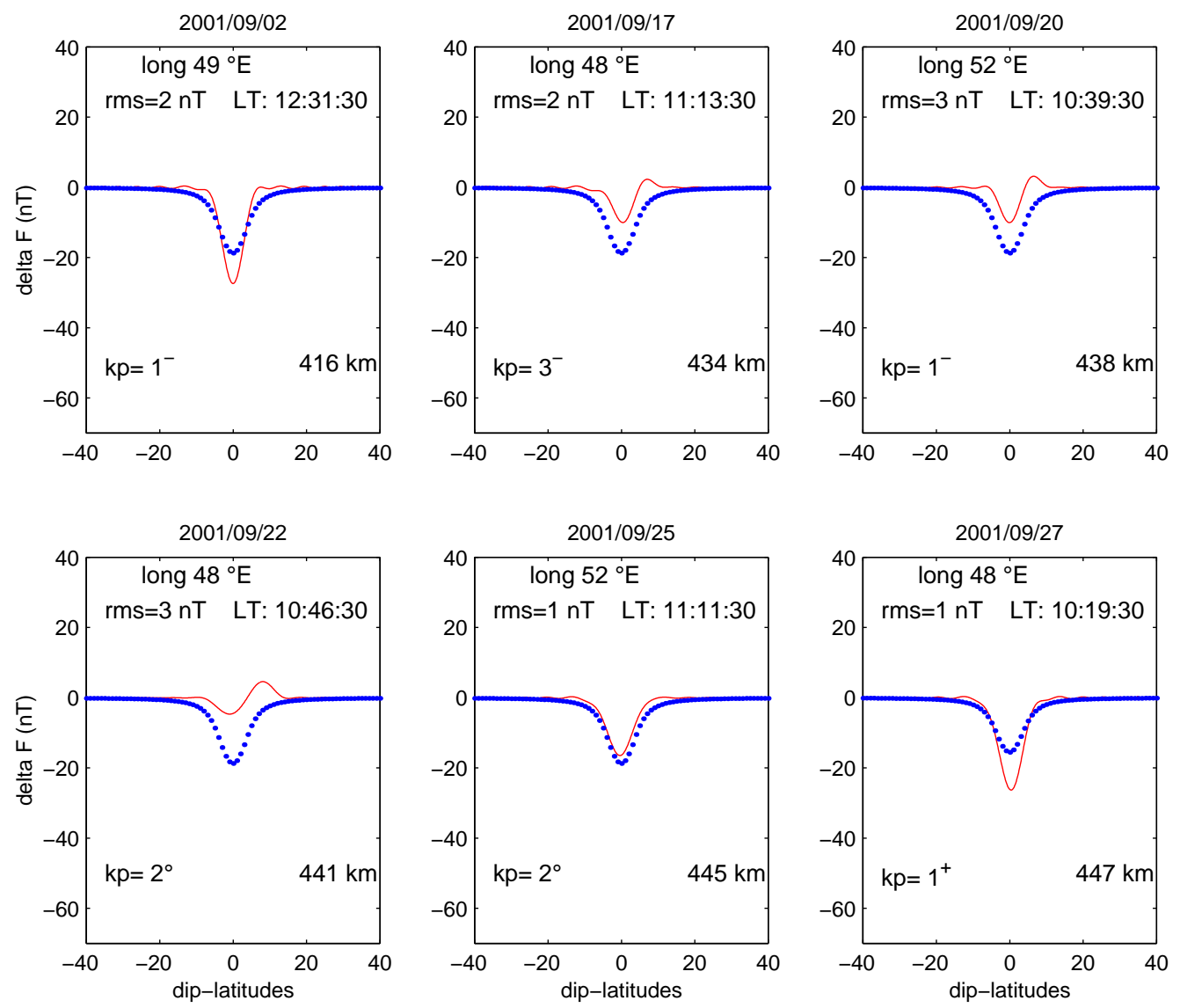

\section{Model EEJ signature \\ Champ EEJ signature}

Fig. 7b. Comparison between the CHAMP satellite magnetic signature and the modeled magnetic effects of the EEJ. The modeled EEJ magnetic effects extrapolated at the altitude, longitude and local time of CHAMP satellite passes (blue dotted line) are compared with the observed EEJ signatures (red solid line) in the longitude band $15^{\circ} \mathrm{W}$ to $30^{\circ} \mathrm{W}$. The modeled profiles were computed using the mean longitudinal profile of the EEJ magnetic signature. The altitude, longitude, local time and the rms are indicated.

components of the electric field. The structure of the groundbased longitudinal profile around noon is globally in good agreement with that obtained from CHAMP scalar magnetic data. The Equatorial Electrojet (EEJ) signatures recorded on board CHAMP satellite have been extracted for the passes closely above West Africa, where a network of three stations has been setup since 1998, for recording the magnetic variations of the EEJ during the $\phi$ rsted and CHAMP satellite missions. The EEJ parameters derived from ground-based magnetic data corresponding to the selected satellite passes have been used to compute the modeled EEJ magnetic signatures at CHAMP altitude $(450 \mathrm{~km})$. The subsequent latitude profiles have been compared to those observed on board the CHAMP satellite. The differences between computed and measured $\Delta F$ peak values are most of the time ( about $69 \%$ of cases) less than $10 \mathrm{nT}$ and the rms are less than $3 \mathrm{nT}$, corresponding to a percentage error of about $10 \%$ with re- spect to the measured EEJ $\Delta F$ peak amplitude. However, on 17 September 2001 and 25 September 2001, the differences between computed and measured peak $\Delta F$ are relatively strong, (e.g. respectively, $32 \mathrm{nT}$ and $42 \mathrm{nT}$ ), with rms of $6 \mathrm{nT}$ corresponding to $28 \%$. The model has overestimated the satellite observed EEJ signatures on 17 September 2001 and 25 September 2001. During these days, enhanced EEJ magnetic effects are observed on the ground $(\Delta H=169 \mathrm{nT}$ and $\Delta H=225 \mathrm{nT}$, respectively). This enhancement is not confirmed by the corresponding overhead observation (Fig. 6). Indeed, on 17 September 2001 and 25 September 2001, the amplitudes of CHAMP borne $\Delta F$ are relatively weak. Since ground-based EEJ parameters were used as input, the equivalent strong current intensity deduced from the ground $\Delta \mathrm{Hs}$ on 17 September 2001 and 25 September 2001 could be the main cause of the model overestimation of the satellite borne EEJ magnetic field during these days. Except on 17 Septem- 


\section{Comparison between the EEJ model and Champ board EEJ signatures}
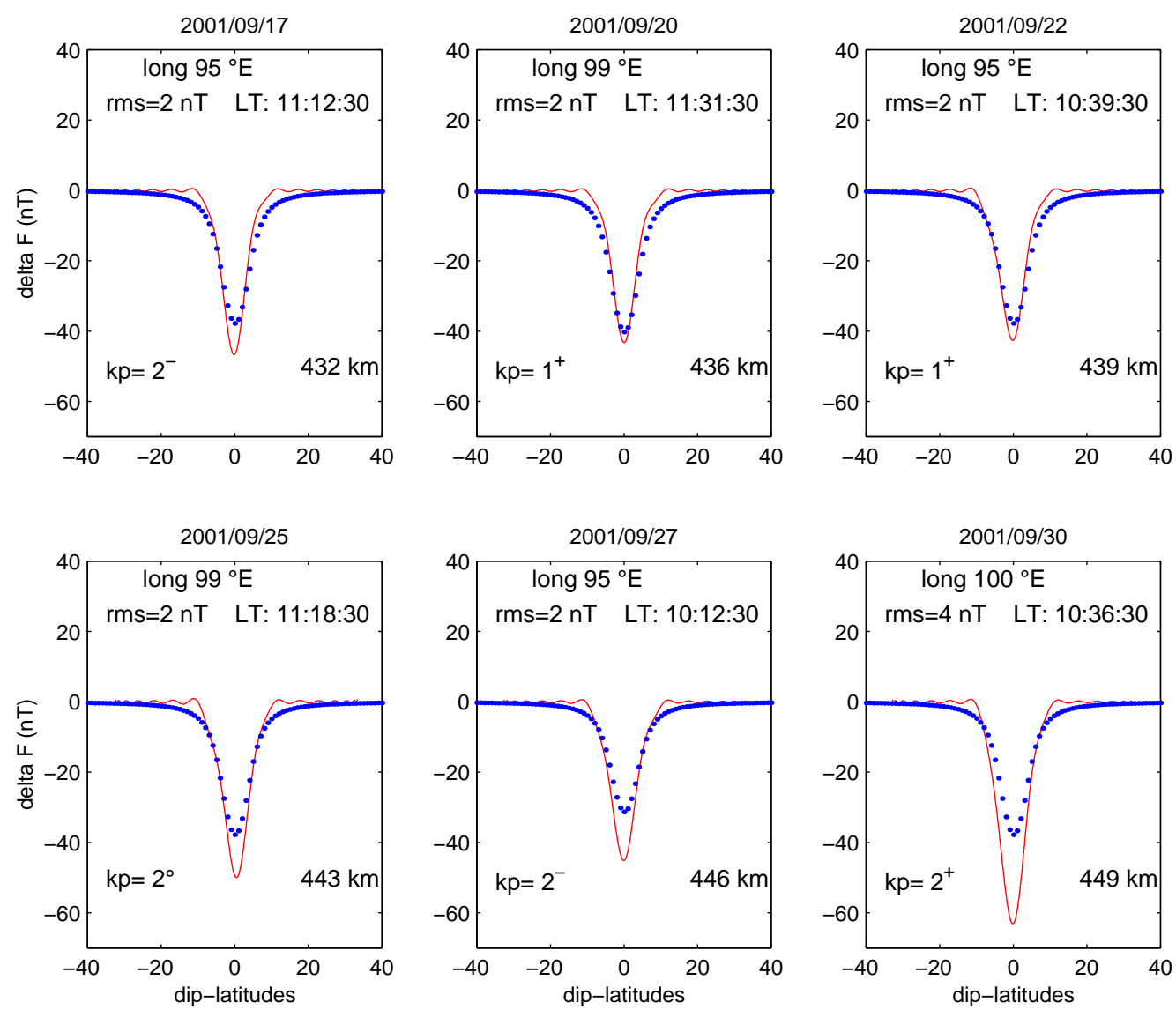

Model EEJ signature
Champ EEJ signature

Fig. 7c. Comparison between the CHAMP satellite magnetic signature and the modeled magnetic effects of the EEJ. The modeled EEJ magnetic effects extrapolated at the altitude, longitude and local time of CHAMP satellite passes (blue dotted line) are compared with the observed EEJ signatures (red solid line) in the longitude band $15^{\circ} \mathrm{W}$ to $30^{\circ} \mathrm{W}$. The modeled profiles were computed using the mean longitudinal profile of the EEJ magnetic signature. The altitude, longitude, local time and the rms are indicated.

ber 2001 and 25 September 2001, the model is generally in good agreement with satellite observation above the West African chain of stations.

Other satellite profiles were selected in every $15^{\circ}$ longitude band. Extrapolating the model to the satellite altitude in those longitude bands, a good agreement was also observed between the model and CHAMP satellite observation, as illustrated by the results in the longitude bands centered, respectively, on $22.5^{\circ} \mathrm{W}, 52.5^{\circ} \mathrm{E}$ and $97.5^{\circ} \mathrm{E}$ (Figs. 7a,b,c).

The $3 \mathrm{EM}$ is aimed to be used for the study and reduction of the EEJ influence on satellite internal magnetic measurements. An EEJ free main field model could be contaminated by the 3EM numerical values of the EEJ magnetic effects. The resulted field could then be inverted in spherical harmonics. This approach corresponds to the case where a main field model, based on satellite data, would contain the magnetic signature of the EEJ.
Despite its quite good estimate of the EEJ magnetic effect observed on board CHAMP satellite, a mean square adjustment should be required to enhance the accuracy of the method.

Acknowledgements. We are grateful to the CNES (Centre National d'Etudes Spatiales) for the financial support, and IRD (Institut pour la Recherche et le Developpement) for magnetic stations that are used in the field experiment in West Africa. We also thank Nahegan Koné and Sylvain Fisher for data acquisition in West Africa, Mihai Roharic for CHAMP data pre-processing, Dr. Minh for providing magnetic data from Vietnam, and Christiane Desfoux for administrative assistance. Dr V. Doumouya received a grant from the French Foreign Ministry (Mission de Cooperation et d'Actions Culturelles à Abidjan) for his stays in France.

Topical Editor M. Lester thanks two referees for their help in evaluating this paper. 


\section{References}

Agu, C. E. and Onwumechili, C. A.: Comparison of the POGO satellite and ground measurements of the magnetic field of the equatorial electrojet. J. Atmos. and Terr. Phys., 43, 801-807, 1981.

Amory-Mazaudier, C., Vila, P., Achache, J., Achy Séka, A., Albouy, Y., Blanc, E., Boka, K., Bouvet, J., Cohen, Y., Dukhan, M., Doumouya, V., Fambitakoye, O., Gendrin, R., Goutelard, C., Hamoudi, M., Hanbaba, R., Hougninou, E., Huc, C., Kakou, K., Kobea-Toka, A., Lassudrie-Duchesne, P., Mbipom, E., Menvielle, M., Ogunade, S. O., Onwumechili, C. A., Oyinloye, J. A., Rees, D., Richmond, A., Sambou, E. Schmuker, E., Tirefort, J. L., and Vassal, J.: International equatorial electrojet year: The African sector, Revista Brasileira de Geofisica, 11, 303-317, Especial, 1993.

Arora, B. R., Mahashabde, M. V., and Kalra, R.: Indian IEEY geomagnetic observational program and some preliminary results, Brazilian J. of Geophys., 11, Eq. (3), 365-384, 1993.

Cain, J. C. and Sweeney, R. E.: The POGO data J. Atmos. and Terr. Phys., 35, 1231-1247, 1973.

Cohen, Y. and Achache, J.: New global vector anomaly maps derived from MAGSAT data. J. Geophys. Res., 95, 10 783-10 800, 1990.

Doumouya, V., Vassal, J., Cohen, Y., Fambitakoye, O., Menvielle, M.: The Equatorial Electrojet at African longitudes: First Results From Magnetic measurement. Ann. Geophys., 16, 658-676, 1998.

Doumouya, V., Cohen, Y., Arora, B. R., and Yumoto, K.: Local time and longitude dependence of the equatorial electrojet magnetic effects, J. Atmos. and Solar-Terr. Phys., 65, 1265-1282, 2003.

Ebun, O.: On the correlation of ground data at Ibadan with POGO satellite results. J. Atmos. and Terr. Phys., 35, 1267-1271, 1973.

Fambitakoye, O. and Mayaud, P. N.: The Equatorial Electrojet and Regular Daily Variation $S_{R}$ : I. A Determination of the Equatorial Electrojet Parameters., J. Atmos. and Terr. Phys., 38, 1-17, 1976a.

Forbush, S. E. and Casaverde, M.: The Equatorial Electrojet in Peru. Carnegie Institut. Washington., Publ. 620, 1961.

Gouin, P.: Correlation of satellite estimates of the equatorial electrojet intensity with ground observations at Addis Ababa. J. Atmos. and Terr. Phys., 35, 1257-1264, 1973.
Kane, R. P.: Comparison of geomagnetic changes in India and the POGO data. J. Atmos. and Terr. Phys., 35, 1249-1252, 1973.

Langel, R. A., Schnetzler, C. C., Philips, J. D., and Horner, R. J.: Initial vector magnetic anomaly map from Magsat. Geophys. Res. Let. 9, 273-276, 1982.

Langel, R. A., Purucker, M. M., and Rajaram, M.: The Equatorial Electrojet and Associated Currents as Seen in MAGSAT Data. J. Atmos. and Terr. Phys. 55, 1233-1269, 1993.

Lühr, H., Maus, S., and Rother, M.: First in-situ observation of night-time F region currents with the CHAMP satellite; Geophys. Res. Let., Vol. 29, No. 10, 1029/2001GL013845, 2002.

Maeda, H., Iymori, T., Araki, T., and Kamie. T.: New evidence of meridional current system in the equatorial ionosphere, Geophys. Res. Let. 9, 243-245, 1982.

Maus, S., Rother, M., Holme, R., Lühr, H., Olsen, N., and Haak, V.: First scalar magnetic anomaly map from CHAMP satellite data indicates weak lithospheric field, Geophysical Research Letters, Vol. 29, No. 14, 10.1029/2001GL013685, 2002.

Onwumechili, C. A. and Agu, C. E.: Longitudinal Variation of the Equatorial Electrojet parameters Derived from POGO Satellite Observations. Planet Space Sciences 29, 627-634, 1980.

Osborne D. G.: Electrojet measurement from satellite and ground. J. Atmos. and Terr. Phys., 35, 1273-1279, 1973.

Ravat D. and Hinze, W. J.: Consideration of variations in ionospheric field effects in mapping equatorial lithospheric Magsat magnetic anomalies. Geophys. J. Int., 113, 387-398. 1993.

Rigoti, A., Chamalaun, F. H., Trivedi, N. B., and Padilha, A. L.: Characteristics of the equatorial electrojet determined from an array of magnetometers in N-NE Brazil, Earth Planet Space 51, 115-128, 1999.

Sabaka, T. J., Olsen, N., and Langel R. A.: A comprehensive model of the quite-time near Earth magnetic field: phase 3, Geophys. J Int., 151, 32-68, 2002.

Yacob, A. and Bhargava, B. N.: The electrojet field from satellite and surface observations in the Indian equatorial region. J. Atmos. and Terr. Phys., 35, 1253-1255, 1973.

Yanagisawa, M. and Kono, M.: Mean ionospheric field correction for Magsat data. J. Geophys. Res., 90, B3, 2527-2536, 1985. 